(Aus der Königlichen Universitäts-Frauenklinik in Tübingen. Director: Prof. Dr. Döderlein.)

\title{
Ueber die Dauererfolge bei der Behandlung der Genital- und Peritonealtuberculose des Weibes.
}

\author{
Von
}

Privatdocent Dr. Karl Baisch.

In älteren Lehrbüchern der inneren Medicin finden wir allgemein die Ueberzeugung, dass die Peritonealtuberculose eine absolut letale Prognose gebe. Es war daher ein wissenschaftliches Ereigniss, als Spencer Wells ${ }^{1}$ ) einen Fall von Peritonealtuberculose durch Laparotomie zur Heilung brachte. Bei einer 22 jährigen Dame fand er ein Abdomen so gross wie am Ende der Schwangerschaft, und constatirte, dass diese Auftreibung durch freie Flüssigkeit verursacht sei. Er diagnosticirte eine subacute Form tuberculöser Peritonitis. Auf interne Behandlung besserte sich der Zustand, später aber trat Verschlimmerung ein, und als Spencer Wells jetzt die Kranke wiedersah, fand er in beiden Weichen tympanitjschen Schall und nur in der Mitte Dämpfung. Ausserdem schien sich bei tiefer Inspiration eine Cyste nach abwärts zu bewegen. Spencer Wells dachte daher an ein Ovariaikystom. Die Laparotomie aber ergab, dass die erste Diagnose die richtige gewesen war, und es sich thatsächlich um tuberculöse Peritonitis gehandelt, hatte. Die Kranke genas, heiratete nach vier Jahren und lebte noch nach 22 Jahren völlig gesund, wenn auch in steriler Ehe.

Erst König ${ }^{2}$ ) freilich hat systematisch die Laparotomie als Heilmittel der Peritonealtuberculose ausgeführt und empfohlen, er

1) Die Krankteiten der Eierstöcke. 1874. S. 103.

2) Centralbl. f. Chir. 1884. No. 6. 1890. No. 35. 
346 Baiseh, Behandlang der Genital- u. Peritonealtuberculose des Weibes.

konnte im Jahre 1890 über 131 Operationen berichten. Seitdem wurden zahllose Fälle von Peritonitis tuberculosa laparotomirt. Neuerdings ist nun aber unverkennbar die frühere Begeisterung für die König'sche Operation gewichen. Man betont die Möglichkeit der spontanen Heilung aller Losalisationen der Tuberculose und erklärt die günstigen Resultate der Chirurgen für Scheinerfolge, die auch ohne Operation eingetreten wären, ja häufig trotz der Operation eingetreten seien.

Der Umschwung zu Gunsten der exspectativen Behandlung knüpft sich an den Namen des Chirurgen Borchgrevink 1). Auch eine Arbeit aus der Döderlein'schen Klinik in Tübingen, von Wunderlich ${ }^{2}$, darf das Verdienst in Anspruch nehmen, durch Hinweis auf die Gefahr postoperativer Kothfisteln zur Kritik gemahnt zu haben.

Borchgrevink verglich 22 laparotomirte Bauchfelltuberculosen mit 22 nicht laparotomirten. Von den Laparotomirten heilten 63 pCt., von den intern Behandelten dagegen 81 pCt. Er nahm an, dass da, wo die Operation Heilung brachte, eine spontane Heilungstendenz vorhanden gewesen war, und daher der Glaube an die Wirkung der Laparotomie lllusion sei.

Die Veröffentlichung Borchgrevinks, die in das Jahr 1901 fällt, gab den Anstoss zu einer Reihe ron Publicationen und hat das grosse Verdienst, die Frage nach der spontanen Heilungsmöglichkeit der Bauchfelltuberculose in lebhaften Fluss gebracht zu haben. Gegenüber einer häufig anzutreffenden Ueberschätzung der Resultate Borchgrevink's muss aber doch hervorgehoben werden, dass seine Therapie keineswegs eine rein exspectativ-interne war, sondern dass er regelmässig die Punction verwendete, sogar im einzelnen Fall zu wiederholten Malen. Auch ist seine Zahl von 22 intern behandelten Fällen zu klein für bestimmte Schlussfolgerungen und es befinden sich darunter zahlreiche Kinder unter 14 Jahren, bei denen die Prognose nach allgemeiner Anschaung eine günstigere ist. Endlich hat Borchgrevink seine Resultate nur 2 Jahre lang controllirt.

Borchgrevink schloss seine Arbeit mit der für einen modernen Chirurgen gewiss ungewöhnlichen Aufforderung, die

1) Borchgrevink, Biblioth. med. Heft 4. 1901.

2) Wunderlich, Ueber die Misserfolge der operativen Behandlung der Bauchfelltuberculose. Dieses Archiv. Bd. 59. S. 216. 
Baisoh, Behandlong der Genital- u. Peritonealtuberculose des Weibes. 347

Chirurgie solle die Peritonealtuberculose der internen Medicin zurückgeben. Man durfte nun gespannt sein, was die Internen an Resultaten mittheilen würden.

Die grösste Zahl intern behandelter Fälle hat Rose ${ }^{1}$ ) aus der Naunyn'schen Klinik pubİicirt. Von 52 intern behandelten und längere Zeit nachbeobachteten Kranken mit Bauchfelltuberculose sind $17=33 \mathrm{pCt}$. geheilt, $34=61 \mathrm{pCt}$. sind gestorben. Das sind also ganz beträchtlich schlechtere Resultate, als Borchgrevink sie erzielt hat. Dort 81 pCt, bei Naunyn nur 33 pCt. Heilungen! Da Rose's Resultate an einem mehr als doppelt so grossen, jahrelang nachbeobachteten Material von über 14 Jahre alten Kranken gesammelt sind, so dürfen wir wohl diese Zahl von 33 pCt. Heilungen - ein Drittel der Erkrankten - als die zur Zeit geltende Heilungschance der Bauchfelltuberculose Erwachsener bei interner Behandlung ansehen.

Leider besitzen wir ausserdem keine grössere Zusammenstellung über die Ergebnisse interner Therapie. Der Versueh, die eigene Erfahrung durch die in der Literatur niedergelegten Berichte zu ergänzen und so eine Statistik über Hunderte ron Kranken zu gewinnen, ist natürlich verschiedentlich unternommen worden. Es ist dieser Weg gerade bei der Tuberculose nicht zweckmässig. Die Kranken sind nach Alter, hereditärer Belastung, individueller Widerstandsfähigkeit, EntwickJungsstadium der Tuberculose und Mitbetheiligung anderer Organe so ausserordentlich versehieden, dass man durch eine Massenstatistik die für den Erfolg einer Therapie massgebenden Factoren mehr verhüllt als aufdeckt. Am förderliehsten ist es, wenn einzelne Kliniken mit grossem Material ihre Resultate unter detaillirter Berücksichtigung aller wichtigen Einzelheiten mittheilen.

Solche Sammelstatistiken, wie sie z. B. Lindner ${ }^{2}$, Roersch ${ }^{3}$, Margarucei ${ }^{4}$ ), Adossides ${ }^{5}$ ) u. A. aufgestellt haben, leiden ausserdem daran, dass die Patienten nicht lange genug nachbeobachtet sind. Mit der Entlassung der Kranken aus der Klinik ist aber ihr Schicksal noch lange nicht entschieden. Es ist für die richtige Einschätzung der Erfolge unserer Tuberculosebehandlung eine jahre-

1) Rose, Grenzgeb. d. inn. Med. u. Chir. Bd. 8.

2) Lindner, Dtseh. Zeitsehr. f. Chir. Bd. 34. 1892.

3) Roersch, Revue de Chirurgie. 1893.

4) Margarucei, Centralbl. f. Chir. 1897. No, 23.

5) Adossides, Inang.-Diss. Halle. 1893. 
348 Baisoh, Behandlung der Genital- u. Peritonealtuberculose des Weibes.

lange Nachbeobachtung mindestens ebenso wichtig, wie z. B. beim Carcinom.

Ich habe unter Berücksichtigung dieser Gesichtspunkte das grosse Material unserer Klinik -- 110 Fälle von Peritoneal- und Genitaltuberculose aus 10 Jahren - in den letzten Monaten einer Nachuntersuchung unterzogen, und von allen Patienten zuverlässige Nachrichten über ihr weiteres Schicksal und jetziges Befinden sammeln können. Weitaus die überwiegende Mehrzahl wurde von mir persönlich untersucht. Auch das ist ein Punkt, der nicht ohne Bedeutung ist. Subjectives Wohlbefinden und objective Heilung deeken sich nicht immer. Bei den tuberculösen Erkrankungen der Genitalorgane finden wir zuweilen ähnlich wie bei den Phthisikern ein völliges Freisein von subjectiven Beschwerden bei ausgedehnter objectiver Erkrankung, die eben nur durch ärztliche Untersuchung festzustellen ist.

Ueber die Frage, wie lange Genitaltuberculosen controllirt werden müssen, bis man ein abschliessendes Urtheil über den Erfolg abgeben darf, sind die Ansichten getheilt. Die meisten Autoren begnügen sich mit 2 Jahren oder noch kürzeren Fristen. Aus unserem eigenen Material vermögen wir nun eine ganz präcise Bestimmung abzuleiten.

Es starben nämlich:

innerhalb der ersten 6 Monate nach Abschluss der

Behandlung. . . . . . . . . . 16 Patienten, im Laufe des 2. Halbjahres . . . . . . . . 7 "

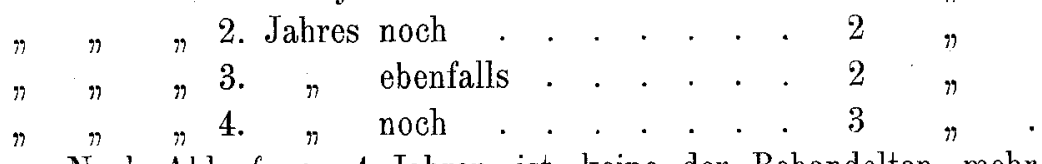

Nach Ablauf von 4 Jahren ist keine der Behandelten mehr gestorben. Ausserdem hat sich aber die bemerkenswerthe Thatsache herausgestellt, dass bei denen, die das 4. Jahr überlebt haben, keine nennenswerthen Beschwerden mehr vorhanden sind und eine Constanz des Befundes eingetreten ist, auch da, wo noch Residuen in Gestalt von Adnextumoren nachgewiesen werden können. Es waren dies einige wenige Fälle, so dass man beinahe sagen kann, nach 4 Jahren sind die Kranken entweder todt oder wieder gesund. Auch von anderen Autoren wird die Dauer der Erkrankung auf 4 Jahre angegeben. So fand Rose nur bei einer Kranken einen Zeitraum von 5 Jahren zwischen Beginn der Erkrankung und Tod. 
Baisch, Behandlung der Genital- u. Peritonealtuberculose des Woibes. 349

Aus der Todtenliste folgt, dass die überwiegende Mehrzahl der Verluste, $5 / 6$, in das erste Jahr fällt und nur $1 / 6$ in die folgenden 3 Jahre. Will man also exact sein, so muss man eine Beobachtungszeit von 4 Jahren einhalten, doch giebt auch schon eine solche von 2 , ja von $1_{1 / 2}^{1 / 2}$ Jahren wenigstens annähernd zuverlässige Resultate.

$\mathrm{Zu}$ den relativ günstigeren Formen gehört die reine

Peritonealtuberculose mit Ascites.

Sie ist in unserem Material mit 38 Kranken vertreten, von denen mehr als die Hälfte im Alter zwischen 15 und 25 Jahren standen. Die jüngste Patientin war 14, die älteste 55 Jahre alt.

Das Hauptcontingent stellt somit das Alter von 15-25 Jahren, dann nimmt die Häufigkeit von Jahr zu Jahr ab.

Der Einfluss der ascitischen Peritonealtuberculose auf dic Menstruation ist ein geringer. In 20 Fällen war die Periode völlig normal, in 8 Fällen etwas zu stark, aber regelmässig. Den 8 Fällen mit verstärktem Blutverlust stehen 6 gegenüber, wo die Periode postponirend auftrat, oder bei früher regelmässiger Periode Amenorrhoe eingetreten war. (2 Fälle von 16- und 18jährigen Mädchen).

In 14 der bisher genannten Fälle war die Periode verspätet, mit 17, 18 und 19 Jahren eingetreten. Es weist dies auf die von Hegar ${ }^{1}$ ) für die Genitaltuberculose betonte gleichzeitige Hypoplasie der Genitalien hin. Eine 14jährige Patientin war noch nicht menstruirt, 2 Erauen mit 52 und 55 Jahren befanden sich bereits in der Menopause, und bei einer Patientin bestand gleichzeitig Gravidität.

Geboren hatten von den verheirateten Frauen alle bis auf 3, die noch nicht lange verheirathet waren. Die Fertilität schwankt zwischen 1 und 12 Kindern and ist abhängig von der Daver der Verheirathung. Sie entspricht durchaus derjenigen gesunder Franen.

Dagegen ist hervorzuheben, dass nach der Erkrankung nur noch eine Patientin geboren hat. Diese eine wurde exspectativ und ohne Punction behandelt. Die Diagnose ist somit nicht absolut sicher. Es folgt aus der Thatsache, dass nur eine einzige Patientin nach der Genesung wieder geboren hat, dass die Peritonealtuberculose so gut wie stets die Fertilität vernichtet. Es ist diese That-

1) Münchener med. Wochenschr. 1899. No. 38. 
350 Baisch, Behandlung der Genital- u. Peritonealtuberculose des Weibes.

sache auch für unser therapeutisches Handeln nicht ohne Bedeutung.

Von den 38 Kranken wurden nur 4 ohne Operation behandelt. Zwei davon sind gestorben, zwei sind gesund geworden, die Zahlen sind aber viel zu klein, als dass man daraus irgend welche statistischen oder therapeutischen Schlüsse ableiten dürfte. Es wurden eben diejenigen exspectativ behandelt, die entweder schon in einem so elenden Zustande waren, dass eine Laparotomie aussichtslos schien - diese sind dann auch nach wenigen Monaten erlegen - oder solche Kranke, bei denen die Flüssigkeitsansammlung und die Beschwerden nicht sehr beträchtlich waren - diese sind gesund geworden.

Bei 34 von 38 Kranken wurde durch Laparotomie oder Colpotomie vom hinteren Scheidengewölbe aus der Ascites entleert.

Es scheint zunächst nicht zu Gunsten der Operationsbehandlung zu sprechen, dass 12 der Operirten, also rund $1 / 3$ gestorben sind. Immerhin ist das Heilungsprocent eben so hoch als bei interner Behandlung die Mortalitätsziffer. Durch chirurgische Behandlung sind $2 / 3$ geheilt, bei Naunyn sind $2 / 3$ gestorben. Ferner aber sind die Erfolge bei den 24 Ueberlebenden so, dass man sie ohne Uebertreibung als sehr gute, zum Theil sogar als glänzende bezeichnen kann.

Bei 15 besteht die Heilung 4-9 Jahre,

$\begin{array}{lllllll}n & 2 & n & n & n & 3 & n \\ n & 4 & n & n & n & 2 & n \\ n & 1 & n & n & n & 11 / 2 & n \\ n & 1 & n & n & n & 1 & n \\ n & 1 & n & n & n & 1 / 2 n\end{array}$

Da wir oben gezeigt haben, dass schon eine 2 jährige $\mathrm{Be}-$ obachtung mit grosser Wahrscheinlichkeit auf definitive Heilung schliessen lässt, so können wir somit in 21 Fällen von Dauerheilung sprechen. Bleiben auch die 3 übrigen gesund, so hätten wir bei den Operirten einen Dauererfolg von 70 pCt. zu verzeichnen.

Es stimmt dieses Resultat gut überein mit den von PapePfannenstiel ${ }^{1}$ ) erzielten 67 pCt. Heilung bei meistens 2 jähriger Beobachtungszeit.

Subjectiv und objectiv gut, d. h. ohne Klagen und Beschwerden, voll arbeitsfähig und ohne jede palpable Veränderung im Abdomen

1) Pape, Beiträge zur Geburtsh. u. Gyn. Bd. VII. S. 422. 
Baisch, Behandlung der Genital- u. Peritonealtuberculose des Weibes. 351

und am Genitale, mit zum Theil recht erheblichen Gewichtszunahmen habe ich 17 von 24 gefunden. 2 versicherten dasselbe brieflich und da die Operation 6-8 Jahre zurückliegt, ist an der definitiven Heilung nicht zu zweifeln. Eine Patientin ist 5 Jahre nach der Operation nach Amerika ausgewandert, eine hat sich verheirathet und nichts mehr von sich hören lassen, aber beides beweist wohl, dass beide Frauen gesund sind, zumal auch hier 9 Jahre seit der Operation verflossen sind.

Man wird gewiss sofort fragen: Verdanken diese Geheilten ihre Heilung lediglich der Operation, wären sie nicht auch durch Punction oder sonstige interne Therapie geheilt worden? Darauf lässt sich im einzelnen Fall eine Antwort überhaupt nicht geben. Die Antwort kann nur durch grosse detaillirte Statistiken interner Kliniken erbracht werden. Zu Gunsten der operativen Behandlung kann immerhin angeführt werden, dass in einzelnen Fällen der Operation eine erfolglose Punction vorausging und dass alle Patienten übereinstimmend angaben, dass vom Tag der Operation ab die Besserung einsetzte und allmählich, wenn auch zuweilen erst nach Monaten zu völliger Genesung führte, ohne dass weitere Eingriffe nöthig: worden.

Warum ist nun bei $2 / 3$ der Kranken Heilung eingetreten, warum ist das dritte Drittel gestorben? Welches sind die Bedingungen, von denen der Erfolg der Operation abhängt? Die Antwort darauf giebt uns zugleich die Anhaltspunkte für die Entscheidung, ob der einzelne Fall der chirurgischen oder internen Behandlung zuzuweisen ist.

Ein Vergleich der Geheilten und Gestorbenen ergiebt, dass von grossem Einfluss

1. die Mitbetheiligung anderer Organe ist, speciell die der Lungen und Pleura. 4 der Gestorbenen litten an Lungen- oder Rippenfell-Tuberculose. Eine davon erlag wenige Wochen nach der Operation unter hohem hectischem Fieber, 3 andere wurden zunächst gesund entlassen, starben aber nach 1 bis 4 Jahren. Polano ${ }^{1}$ ) hat mit besonderem Nachdruck auf die Gefahr gleichzeitiger Lungenaffectionen hingewiesen. Nach unseren Erfabrungen sind es übrigens gerade Spätverluste, die durch die Complication mit Lungentuberculose verursacht werden und streng genommen kann man dies nicht gegen die Zweckmässigkeit der Operation anführen.

1) Polano, Zeitschr. f. Geburtsh. u. Gynäk. 1901. Bd. 44. S. 85. 
352 Baisch, Behandlung der Genital- u. Peritonealtuberculose des Weibes.

Die Operation hat immerhin die Bauchfellentzündung günstig beeinflusst und dẹn Kranken für Jahre Besserung gebracht. Ueberhaupt dürfen leichtere Spitzenaffectionen nicht ohne weiteres als Contraindicationen gegen die chirurgische Behandlung angesehen werden: bei 7 unserer dauernd Geheilten bestand zur Zeit der Operation eine objectiv nachweisbare Lungenaffection und Husten. Es ist ja auch durchaus verständlich, dass eine gründliche und dauernde Entfernung des Ascites durch Erleichterung der Athmung von Vortheil sein muss.

In zweiter Linie ist unter den für den Erfolg maassgebenden Factoren Fieber vor der Operation zu nennen. Es ist gewiss kein Zufall, dass von unseren $7 \mathrm{im} 1$. Vierteljahr nach der Operation Gestorbenen 3 fieberten, während von den 24 Geheilten keine länger anhaltende und höhere Temperatursteigerungen hatte. Nur 3 hatten je einmalige Steigerung bis höchstens $38^{\circ} \mathrm{C}$. und gerade diese 3 machten eine schwere fieberhafte Reconvalescenz durch, wenn sie auch schliesslich dauernd geheilt wurden. Borchgrevink hat der Laparotomie ganz besonders den Vorwurf gemacht, dass nach ihr Fieber auftrete und dieses auf die Verkäsung der Tuberkel hindeute, die eben durch den schwächenden Einfluss der Operation auf den Organismus begünstigt werde. Für fiebernde Tuberculöse ist in der That ein schädigender Einfluss der Operation nicht zu verkennen und auch wir müssen nach unseren Erfahrungen mehrtägiges, wenn auch niedriges Fieber entschieden für eine Gegenanzeige gegen einen chirurgischen Eingriff halten. Jedenfalls wird die Prognose dadurch sehr getrübt.

Solche subfebrile Temperaturen deuten auf einen progredienten Character der Erkrankung, der dann auch darin zum Ausdruck kommt, dass zuweilen nach der Laparotomie die Tuberculose auf die Wunde übergreift, sie zu käsigem Zerfall bringt und die so sehr unangenehmen Kothfisteln entstehen. Wir haben 3 Patienten auf diese Weise verloren.

Wenn sich solche Fisteln nach der Laparotomie bilden, so ist es nur ein schwacher Trost, dass Kothfisteln nicht selten auch spontan ohne vorausgegangenen chirurgischen Eingriff entstehen.

Bei den fiebernden Kranken trägt die Tuberculose einen ausgesprochen malignen Charakter. Ganz ähnlich bösartig verläuft sie bei Kranken, deren Körper sonstwie geschwächt ist, z. B. bei Herzfehlern oder bei Gravidität und im Puerperium. Wir verloren eine Patientin mit Vitium cordis und eine weitere Patientin, bei der 
Baisch, Behandlung der Genital-, u. Peritonealtuberculose des Weibes. 353

gleichzeitig Schwangerschaft im 6. Monat und Peritonealtuberculose bestand.

Die besten Erfolge hat man somit bei Kranken, die nicht fiebern, die keine anderweitige schwere Localisation der Tuberculose darbieten und kräftig genug sind, um einen operativen Eingriff nicht als eine ernstliche Schwächung des Organismus zu empfinden. So lagen die Verbältnisse bei unseren glatt und dauernd geheilten Kranken. $O b$ man freilich bei solch günstigen Vorbedingungen nicht auch mit der Punction allein, eventuell der wiederholt ausgeführten Punction bei gleichzeitiger kräftigender Allgemeinbehandlung und Bettruhe, ebenso günstige Resultate erzielen wird, ist eine Frage, die uns die interne Behandlung beantworten muss.

Es wäre dies sehr zu begrüssen, denn die Laparotomie hat eben zweifellos gewisse unvermeidbare Nachtheile. Auch wenn wị von der Narkose absehen, die wir ja durch Lumbalanästhesie ersetzen können, so resultirt doch eine Bauchwunde, die die freie Athmung hindert und die Expectoration erschwert, die ferner zu Kothfisteln und später zu. Hernien disponirt. Ein Vorschlag von Condamine, den in Deutschland Löhlein sehr unterstützt hat, umgeht diese Gefahr und bietet noch weitere Vortheile vor der Laparotomiewunde. Diese Autoren haben empfohlen, die Peritonealhöhle vom hinteren Scheidengewölbe aus zu eröffnen. Sie. haben mit dieser Empfehlung vielseitig Anklang gefunden ${ }^{1}$ ). Vom tiefsten Punkt der Bauchhöhle aus fliesst der Ascites noch weit vollständiger $a b$, als durch die Bauchwunde. Man kann ferner durch Einlegen eines T-förmigen Gummidrains dafür sorgen, dass auch in den nächsten Tagen noch sich etwa neu bildender Aseites sofort abgeleitet wird. Vor Allem aber sieht man darnach keine Kothfisteln entstehen. Wir haben diese Methode 11 mal verwendet, auffallend häufig haben wir aber darnach während der Reconvalescenz höhere Temperatursteigerungen auftreten sehen, weit häufiger als bei der Laparotomie. Die Ursache davon ist nicht ganz klar, möglicher Weise kommt es zur Ascendenz von Keimen aus der Scheide und zu Mischinfection.

Winter ${ }^{2}$ ) und Pfannenstiel ${ }^{3}$ ) haben den Rath gegeben,

1) Baumgart, Deutsche med. Wochenschr. 1901. No. 2-4. S. 177.

2) Winter, Deutsche med. Wochenschr. 1902. No. 23. Vereinsbeil.

3) Pape, Hegar's Beiträge z. Geburtsh. u. Gyn. 1903. Bd. 7. S. 432. 
354 Baisch, Behandlung der Genital- u. Peritonealtuberoulose des Weibes.

man solle stets, wenn man das Abdomen öfine, auch die Tuben mit entfernen; und Rose und v. Krencki ${ }^{1}$ ) haben statistisch nachzuweisen versucht, dass die Patientinnen nach Entfernung der Tuben günstigere Aussichten für Dauerheilung haben. Aus unserem eigenen Material ist ein wesentlicher Einfluss der Belassung oder Entfernung der Tuben auf die Dauerergebnisse nicht abzuleiten. Zwei Patientinnen wiesen 5 Jahre nach der Operation bei der Untersuchung einen kleinen Adnextumor der einen Seite auf, bei einer Patientin hat er sich nach weiteren zwei Jahren zurückgebildet.

Da, wie wir oben gesehen haben; die Tuberculose die Fertilität so gut wie immer vernichtet, ist gegen eine Entfernung der Tuben an sich gewiss nichts einzuwenden und wo es ohne weitere Complication der Operation geschehen kann, nur zweckmässig zu nennen.

Wesentlich ungünstiger als bei der ascitischen form liegt nun die Prognose bei der

trockenen adhäsiven Peritonealtuberculose.

Ganz scharf lassen sich freilich diese beiden Formen nicht trennen, es finden sich alle Uebergänge, so dass es zuweilen etwas willkürlich ist, wenn man einen Fall zu dieser oder jener Kategorie rechnet.

Unsere 22 Kranken mit trockener Bauchfelltuberculose standen im Alter zwischen 15 und 52 Jahren. Die überwiegende Mehrzahl, 15 davon, waren we die der ascitischen Form zwischen 15 und 25 Jahre alt. Im Alter zwischen 25 und 35 Jahren standen 4, zwischen 35 und 45 Jahren noch 6 Kranke.

Die Menstruation war bei 2 Patientinnen noch nicht eingetreten, obwohl sie schon 16 und 19 Jahre alt waren. Bei 4 hatte sie bereits wieder aufgehört. Diese letzten waren 41, 44, 50 und 52 Jahre alt. Völlig normal war sie nur bei einem kleinen Bruchtheil, bei 6 Kranken. Bei einer weiteren Patientin war sie besonders schwach. Bei 7 war sie nach Typus, Dauer und Stärke ohne Abnormität, hatte sich aber relativ spät eingestellt, erst zwischen dem 16. und 19. Lebensjahre. Auch 3 weitere Kranke zeigten verspäteten Eintritt der Pubertät, hatten aber verstärkten Blutverlust. Ueber Unregelmässigkeit und verlängerte Dauer der Intervalle klagte eine Patientin.

1) v. Krencki, Inaug.-Diss. Königsberg. 1902. 
Baisch, Behandlung der Genital- u. Peritonealtubereulose des Weibes. 355

Einen directen Einfluss der Erkrankung auf die Menstruation wiesen 5 Kranke auf, und zwar hatte bei 3 die Periode seit 5 bis 7 Monaten cessirt, bei 2 war sie seit der Erkrankung viel stärker geworden.

Es besteht somit keine einheitliche Einwirkung der Erkrankung auf den Ablauf der Menstruation, und ein typisehes Blutungsbild existirt nicht. Bemerkenswert ist bei der trockenen Form wie bei der ascitischen die Häufigkeit, mit der die Pubertät sich verspätet einstellte, nämlich in 12 von 22 Fällen. Wir machten bereits darauf aufmerksam, dass dies für den von Hegar betonten Zusammenhang zwischen Tuberculose und Entwicklungsstörung spricht.

Die Fruchtbarkeit der Patientinnen war vor ihrer Erkrankung durchaus nicht ungünstig. Von 14 verheiratheten Frauen wurden im Ganzen 63 Kinder geboren.

Die Zahl der Kinder steht bei allen in Proportion zur Dauer der Ehe, nur bei einer einzigen bestand seit 5 Jahren Sterilität, nachdem sie ein Kind geboren hatte.

Nach der Erkrankung baben auch die Genesenen nie mehr concipirt, und es vernichtet somit auch die adhäsive Bauchfelltuberculose wie die ascitische die Fortpflanzung der Frau.

Von 22 Fällen mit keinem oder nur spärlichem Ascites ist die Hälfte gestorben; und zwar sind von 11 exspectativ behandelten Kranken 8 gestorben, von 11 Operirten 8 gesund und arbeitsfähig geworden. Von den Genesenen sind 5 Patientinnen 5 Jahre und länger in Beobachtung.

Ist es schon bei der ascitischen Form nicht leicht, den Werth der Laparotomie richtig einzuschätzen, so ist es noch viel schwieriger, bei der trockenen Form zu sagen, ob und welches Verdienst die Laparotomie an der Heilung hat. Man kann sich eben noch weit weniger als bei der exsudativen Form eine exacte Vorstellung davon machen, worin eigentlich der Nutzen der Laparotomie dabei gelegen sein soll. Es sind nurProbelaparotomien und Probecolpotomien. Das Abdomen wird, sobald die Diagnose gestellt ist, sofort wieder geschlossen. Und das ist noch das Beste, was man thun kann. Fängt man an, Darmverwachsungen zu lösen, so dauert es gar nicht lange und der enorm brüchige Darm reisst ein. Nähte halten nicht, wenigstens nicht auf die Dauer, und es entsteht wohl ganz regelmässig eine Kothfistel. Wir haben eine Patientin durch Inanition an einer solchen Kothfistel verloren. 
356 Baisch, Behandlung der Genital- a. Peritonealtuberculose des Weibes.

Wenn man allerdings nur die Zahlen hört, dass von 11 Operirten 8 gesund geworden sind, so scheint das sehr zu Gunsten der Operation zu spechen. Sieht man sich aber die Fälle im einzelnen genauer an, so wird man doch wieder zweifelhaft. So haben 2 Patienten nach der Operation Kothfisteln bekommen, die eine sogar eine Bauchdecken- und eine Scheidendünndarmfistel, beide lagen wochen- und monatelang nach der Operation fiebernd und elend zu Bett, beide sind heute - die eine 10, die andere 4 Jahre seit der Entlassung - vollkommen arbeitsfähig, sehen blühend aus, und die eine hat sich einstweilen verheirathet. Bei beiden haben sich die Fisteln vollkommen geschlossen. Hier ist man entschieden versucht zu sagen, dass sie nicht durch die Operation, sondern trotz der Operation genesen sind. Hier besass der Organismus die Kraft, nicht nur die Krankheit zu überwinden, sondern auch die Laparotomie, die durch Erzeugung der Kothfisteln gewiss nicht zur Kräftigung des Körpers beigetragen hatte.

Verblüffend sind freilich wieder andere Fälle, wo nach längerem Zuwarten bei fortschreitender Verschlimmerung die Laparotomie doch noch Heilung brachte. So fanden sich bei einer 33 jährigen Frau, 1 Jahr nach der ersten Untersuchung, überall auf dem Peritoneum bis erbsengrosse Tuberkel, der Darm war in ein unlösbares Convolut verbacken, Uterus und Adnexe unter sich und mit der Umgeburg zu einem grossen Tumor verschmolzen. Nach Constatirung dieses Befundes wurde das Abdomen sofort wieder geschlossen. Jetzt, $1 \frac{1}{2}$ Jahre nach der Entlassung, ist die Patientin voll arbeitsfähig, sieht blühend aus, das Abdomen ist weich, ohne jede Resistenz und Druckempfindlichkeit, und nur rechts neben dem Uterus fühlt man einen kleinen unempfindlichen Tumor.

Ob freilich an diesem Erfolg die längere Bettruhe und die Pflege in der Klinik nicht ebenso viel Antheil haben wie das Oeffnen. und Schliessen des Abdomens, ist schwer zu sagen. Gerade in dem letzt beschriebenen Fall liegt diese Vermuthung um so näher, als die Patientin, eine Wirthsfrau, sich zu Hause nicht schonen konnte.

Die Laparotomie wird von vielen und nambaften Autoren, Merkel ${ }^{1}$ ), Condamine, Margarucci, Jordan ${ }^{2}$ ), H. W. Freund ${ }^{3}$, u. A. auch bei trockener, adhäsiver Peritonealtuberculose warm

1) Merke1, Zeitschr. f. Gehurtsh. u. Gyn. 1898. No. 3.

2) Jordan. Brun's Beiträge zur Chir. Bd. 13.

3) H. W. Freund, Hegar's Beiträge z. Geburtsh. u. Gyn. Bd. VII. H.3. 
Baisch, Behandlung der Genital- u. Peritonealtuberculose des Weibes. 357

empfohlen. Auch Lauper ${ }^{1}$ ) in der Kocher'schen Klinik hat in einigen wenigen Fällen gute Erfahrungen damit gemacht. Gerade bei dieser Form wäre die Mittheilung der Resultate einer systematischen internen Therapie ganz besonders erwünscht.

Von unseren eigenen, exspectativ behandelten 11 Kranken haben wir 8 verloren. Es liegt sehr nahe, diese hohe Mortalität gegen die exspectative Therapie zu verwerthen. Mit Unrecht. Es waren entweder sehr vorgeschrittene Fälle mit inoperablen Beckenexsudaten oder hektischem Fieber - diese sind in wenigen Monaten zu Grunde gegangen oder es waren Kranke mit ernsteren Lungenaffectionen, bei denen die Operation sich schon allein dadurch verbot und diese sind nach 3-4 Jahren gestorben.

Wesentlich anders nun und viel leichter zu beurtheilen sind die Verhältnisse bei denjenigen Patienten, bei denen die

tuberculöse Tubenerkrankung

in Gestalt der Salpingitis oder ihres Endstadiums, der Pyosalpinx, im Mittelpunkt des klinischen Bildes steht.

Auch hier haben wir übrigens kein einheitliches Krankheitsbild vor uns. Bald ist die Adnexerkrankung mit einer PeritonealTuberculose combinirt, die verschieden stark ausgebreitet sein kann, bald sind nur die Tuben selbst ergriffen; und auch bei der reinen Adnextuberculose finden sich alle Grade und Entwicklungsstadien.

Insgesammt sind es 45 Fälle von vorwiegender. Tubentuberculose, von denen 14 zwei Jahre und länger, 3 vier Jahre und darüber in Nachbeobachtung stehen. Auch hier ist es wieder das Alter zwischen 15 und 25 Jahren, das mit 20 Kranken das Hauptcontingent stellt. Im Alter zwischen 26 und 35 Jahren standen 19 Patientinnen, zwischen 36 und 45 Jahren 5 , und je eine Kranke war 50 und 63 Jahre alt.

Die Menstruation war nur bei 14 Patientinnen völlig regelmässig, bei 11 war sie verstärkt und 3 litten an ausgesprochenen Metrorrhagien.

Wie bei den reinen Peritoneal-Tuberculosen ist auch bei der Tubentuberculose der verspätete Eintritt der Pubertät auffallend, insofern bei 22 Kranken die Periode erst nach dem 16. Lebensjahr, bei einigen erst mit dem 20. Lebensjahr eintrat. 2 Kranke befanden sich bereits in der Menopause.

1) Làuper, Deutsche Zeitschr. f. Chir. Bd. 59. 1901. 
358 Baisch, Behandlung der Genital- u. Peritonealtuberculose des Weibes.

Unter dem Einfluss der Erkrankung war die früher regelmässige Periode bei 6 Patientinnen seit einem bis dreiviertel Jahren ausgeblieben.

Es besteht somit auch bei der Tubentuberculose kein constanter und typischer Einfluss der Erkrankung auf den Ablauf der Menstruation. Es findet sich sowohl der normale Typus, wic auch die Verstärkung, und ihr Gegentheil, die völlige Amenorrhoe.

Die Fruchtbarkeit der Patientinnen war vor der Erkrankung nicht beeinträchtigt. Je nach der Dauer der Verheirathung schwankt. sie zwischen 1 und 7 Kindern. Nor eine Patientin ist seit 5 Jahren steril verheirathet.

Dagegen hat nach der Erkrankung keine Patientin mehr geboren.

Von unseren 45 Patientinnen wurde $1 / 4$ (13) ohne Operation, $3 / 4$ (32) chirurgisch behandelt.

Von den exspectativ behandelten 13 Fällen sind 8 gestorben, und es wiederholt sich hier die frühere Beobachtung: Die eine Hälfte sind schwere, weit fortgeschrittene, operativ nicht inehr anzugreifende Erkrankungen, die im ersten halben Jahr nach der Entlassung zum Exitus kamen. Bei der anderen Hälfte fand sich von vornherein noch Lungen- oder Pleuratuberculose, oder sie gesellte sich nachher dazu.

Aus diesen Fällen die Unzulänglichkeit der exspectativen Behandlung beweisen zu wollen, wäre natürlich ganz verkehrt. Weit mehr spricht gegen die spontane Heilungstendenz der Tubentuberculose der Verlauf bei 2 Patientinnen, die an sich beide sehr wohl zur Operation geeignet gewesen wären, diese aber verweigerten. Sie sind, die eine nach 2 , die andere nach $1 \frac{1}{2}$ Jahren gestorben.

Immerhin sind von den 13 zuwartend behandelten Patientinnen bei der Nachuntersuchung 5 als gebessert befunden worden; aber das Resultat war in diesen Fällen doch ein recht unvollkommenes.

Bei 4 Patientinnen nämlich bestanden nach wie vor faustgrosse Adnextumoren zu beiden Seiten des Uterus. Trotzdem ist subjectiv eine wesentliche Besserung za verzeichnen. Es erfolgte Gewichtszunahme in einem Falle bis zu 29 Pfund, und auch die Arbeitsfähigkeit ist nahezu vollkommen zurückgekehrt.

Nur eine Patientin ist nicht nur subjectiv beschwerdefrei, anch der objective Befund ist ein vollkommen normaler. Die vor 6 Jahren vorhandenen doppelseitigen, allerdings kleinen Adnextumoren sind nicht mehr nachzuweisen. Es handelt sich um ein 
Baisch, Behandlung der Genital- ט. Peritonealtuberculose des Weibes. 359

24 jähriges Bauernmädchen, das 2 Jahre vor der ersten Aufnahme eine Pleuritis überstanden hatte.

Sehr beeinträchtigt wird ferner der Werth der exspectativen Behandlung durch alle die Erlebnisse, wo nach anfänglich interner Therapie doch noch zum Messer gegriffen werden musste. Wir verzeichnen 4 solcher Fälle. In allen musste wegen zunehmender Verschlimmerung nach $1 \frac{1}{2}-4$ Jahren operirt werden, und bei allen ist nunmehr vollkommene Heilung eingetreten, die bei dreien $11 / 2-3$ Jahre besteht. Der Genitalbefund ist normal, die Beschwerden sind verschwunden, das Körpergewicht hat zugenommen, und es ist volle Arbeitsfähigkeit eingetreten.

Von diesen Fällen sind 3 von uns exspectativ behandelt worden, während die Operation nachträglich von anderer Seite vorgenommen wurde.

Den 13 exspectativ behandelten Fällen stehen nun 32 operirte gegenüber. 23 Patientinnen, das sind $2 / 3$, sind gesund und arbeitsfähig geworden.

9 Patientinnen allerdings sind gestorben, und zwar 4 davon primär; 3 an Peritonitis nach Rectumverletzungen, die nach Lösung von Adnextumoren sich ereigneten, eine an Bronchopneumonie. Die anderen 5 erlagen zu Hause nach 3 Monaten bis $2 \frac{1}{2}$ Jahren. Dabei ist hervorzuheben, dass eine dieser Kranken, die nach drei Monaten starb, an Lungentuberculose litt, und dass bei den anderen 4 Kranken bei der Operation nur die Adnexe der einen Seite entfernt worden waren. Diese letzteren starben nach $1-1 \frac{1}{2}$ Jahren.

Bei 13 von den 25 Operirten und noch Lebenden ist das Resultat ein vollkommenes, insofern objectiv und subjectiv voller Erfolg eingetreten ist. Bei 11 davon liegt die Operation mehr als 2 Jahre zurück, bei 6 Patientinnen sogar 4-8 Jahre.

Objectiv sehr gut, dagegen subjectiv unvollkommen ist der Erfolg bei 4 weiteren Kranken, von denen 1 an Diarrhoen, 1 an Husten, 1 an Ausfallserscheinungen und 1 an Melancholie lepidet. Diese Beschwerden fallen entweder gar nicht oder nur sehr indirect der Operation zur Last.

In 6 Fällen dagegen ist zwar der subjective Erfolg ein guter, sofern keine Beschwerden mehr bestehen und die Arbeitsfähigkeit voll zurückgekehrt ist, dagegen sich aufs neue Tumoren entwickelt haben. Diese Fälle sind nun von ganz besonderem Interesse, sie geben uns für die Wahl.der Operationsmethode die werthrollsten Anhaltspunkte. 
360 Baisch, Behandlung der Genital- u. Peritonealtuberculose des Weibes.

Nur 3 dieser Fälle nämlich sind radical operirt worden, und gerade bei ihnen ist der Befund ein annähernd normaler. Es finden sich nur kleine Resistenzen, von denen nicht mit Sicherheit gesagt werden kann, ob sie alte Stumpfexsudate oder Scheintumoren, bestehend aus verwachsenen Darmschlingen, sind. In einem Falle liegt der gänseeigrosse Tumor hoch oben an der Linea terminalis und stellt wohl ein tuberculöses Lymphdrüsenpaket dar. Bei der Operation waren Uterus und Adnexe entfernt worden.

Gerade diese 3 Frauen sind übrigens arbeitsfähig, haben an Gewicht zugenommen und sind frei von Beschwerden.

In den anderen 3 Fällen dagegen sind nur unvollkommene Operationen ausgeführt worden. In einem Fall eine Probelaparotomie mit Zurücklassung der unlösbar verwachsenen Adnextumoren. Hier finden sich jetzt doppelseitige grosse Tubensäcke. Im zweiten Falle wurde nur Colpotomie vorgenommen und Ascites entleert, während die verwachsenen, in dicke, unbewegliche Tumoren umgewandelten Adnexe zurückblieben. Auch hier ist jetzt links ein faustgrosser, rechts ein kleinerer Adnextumor zu palpiren. Im 3. Falle endlich wurde nur eine Tube entfernt, jetzt findet sich auf der andern Seite eine orangegrosse Adnexgeschwulst.

Auch in diesen Fällen ist mérkwürdiger Weise der subjective Erfolg ausgezeichnet und die Arbeitsfähigkeit ist eine so vollkommene, dass einer der Patientinnen die Invalidenrente entzogen wurde.

Ueberblicken wir diese Fälle subjectiv oder objectiv unvollkommener Heilung, so illustriren sie am deutlichsten die relativ geringe spontane Heilungstendenz der Tubentuberculose. Die Gestorbenen oder nicht vollkommen Geheilten sind diejenigen, bei denen eine Tube zurückgelassen wurde, entweder weil ihre Entfernung wegen intensiver Verwachsungen technisch unmöglich war, oder weil sie gesund zu sein schien. Nicht einmal mit den Anfangsstadien der Erkrankung, die makroskopisch noch gar nicht erkannt werden können, ist der Organismus aus eigener Kraft fertig geworden. Die zurückgelassenen Tuben haben sich zu Pyosalpinxsäcken ausgebildet, die theils den Kranken später doch noch das Leben kosteten, theils nachträglich durch eine zweite Operation entfernt werden mussten, im besten Falle aber durch ihr Weiterbestehen eine ständige Gefahr für ihre Trägerin darstellen.

Die Tuben gehören zu denjenigen Organen des Körpers, die mit Vorliebe von der Tuberculose befallen werden und ihr gegen- 
Baisoh, Behandlung der Genital- u. Peritonealtuberculose des Weibes. 361

über eine ganz besonders geringe Widerstandsfähigkeit besitzen. Daraus erklären sich die ungünstigen Erfahrungen bei der Zurücklassung einer Tube. Andererseits ist bei der Localisation der Tuberculose in einem so wenig wehrkräftigen Organ der Versuch, den Körper in seinem Kampfe gegen die Krankheit durch Entfernung des isolirten Herdes zu unterstützen, weit aussichtsvoller und die günstige Wirkung weit verständlicher als bei der diffusen Peritonealtuberculose.

Unsere Erfahrungen sprechen ganz entschieden für die Forderung, in jedem Fall beide Tuben zu entfernen, auch wenn die eine scheinbar ganz gesund ist. Eine andere Frage ist, ob man stets auch Uterus und Ovarien exstirpiren soll. Hegar ${ }^{1}$ ), Sellheim ${ }^{2}$ ) und Veit ${ }^{3}$ ) haben den Satz aufgestellt, dass, wenn man bei der Tuberculose überhaupt operire, man möglichst radical operiren müsse. Aus unserem eigenen Material lässt sich die Nothwendigkeit, stets auch den Uterus und beide Ovarien zu entfernen; nicht ableiten. Keine der Patientinnen, bei denen der Uterus' zurückgelassen wurde, hat ein Recidiv bekommen. Wir wissen durch die Experimente von Baumgar.ten ${ }^{4}$ ), dass die Genitaltuberculose ein descendirender Process ist. Nach der Entfernung der Tuben fällt die beständige Impfung mit virulentem Secret fort, und das zur Tuberculose wenig disponirte Organ vermag alsdann gesund zu bleiben oder wieder gesund zu werden. Freilich wird man nicht selten, schon um einfachere Wundverhältnisse zu schaffen, den Uterus zu entfernen genöthigt sein. Immer aber empfiehlt es sich, die Tubenecken keilförmig zu excidiren.

Besonders schwer wird die Entscheidung, ob man auch beide Ovarien exstirpiren soll, seitdem wir durch von Franqués ${ }^{5}$ ) und Polano's Untersuchungen wissen, dass die Ovarialtuberculose nicht so ganz selten ist. Handelt es sich doch meist um Patientinnen in jugendlichem Alter, bei denen die Entfernung beider Keimdrüsen die schwersten Ausfallserscheinungen zur Folge haben kann. Auch

1) Hegar, Die Entstehung, Diagnose u. chirurg. Behandlung der Genitaltuberculose des. Weibes. Stuttgart. 1886.

2) Sellheim, Hegar's Beiträge. Bd. VI. S. 406.

3) Veit, Internat. Congress f. Geburtsh. u. Gyn. Rom. 1902. S. auch Monatsschr. f. Geburtsh. u. Gyn. Bd. XVI. Ergänzungsheft.

4) Baumgarten, Berl. klin. Wochenschr. 1904. No. 42.

5) Die Königl. Univ.-Franenklinik in Wärzburg. Berichte und Studien. Stuttgart. 1903. S. 84. 
362 Baisch, Behandlung der Genital- u. Peritonealtubereulose des Weibes.

wir haben bei 2 Kranken solche ernste, den Erfolg der an sich gelungenen Operation stark trübende Ausfallserscheinungen eintreten sehen.

$\mathrm{Da}$ wir nun nur in einem einzigen Falle nach Zurücklassung eines Ovariums ein Recidiv beobachtet haben in Gestalt eines stark kinderfaustgrossen Tumors der betreffenden Seite, der jedoch das subjective Wohlbefinden und die Erwerbsfähigkeit nieht beeinträchtigte, so möchten wir doch bei jugendlichen Patientinnen unter 30 Jahren sehr für die Zurücklassung wenigstens eines Ovariums plädiren.

Als Operationsweg empfiehlt sich in erster Linie die Laparotomie, die ein exactes Operiren, Schonung der Organe, vorsichtige Lösung der Adhäsionen, Vermeidung von Nebenverletzungen, reinliche Versorgung des Operationsfeldes und eventuell ein conservatives Vorgehen gestattet. Nur bei Fehlen festerer Adhäsionen ist der vaginale Weg einfacher und ermöglicht eine raschere Reconvalescenz.

Von unseren sämmtlichen 110 Fällen von Bauehfell- und Genitaltuberculose sind 40 gestorben, entweder primär oder im Laufe der nächsten 4 Jahre. Das giebt eine Gesammtmortalität von $36 \mathrm{pCt}$. Diese Resultate sind weit besser als der Durchschnitt der internen Therapie. Wir hätten darnach eigentlich allen Grund, mit der operativen Behandlung der Bauchfell- und Genitaltuberculose zufrieden zu sein. Trotzdem ist ihre Ueberlegenheit über die interne Therapie nicht zweifellos erwiesen. Vor allem kamen eben nur in ganz vereinzelten Fällen bei unseren Kranken alle die Heilfactoren in Anwendung, über die heutzutage die innere Medicin bei der Tuberculose verfügt. Leider ist eine solche Behandlung für wenig bemittelte Kranke sehr theuer, weil sehr langwierig; und für Kassenmitglieder ist sie aus dem Grunde nicht leicht durchführbar, weil wohl die Lungentuberculösen grosse und wohleingerichtete Sanatorien zur Verfügung haben, für die übrigen Formen der Tuberculose aber noch nicht ausreichend gesorgt ist. Bis hierin Wandel geschaffen sein wird, werden wir wohl im Interesse der raschen Genesung unserer Kranken häufig den operativen Weg einschlagen müssen. Systematische Nachuntersuchungen aber müssen uns die Gesichtspunkte erkennen lassen, nach denen wir die Fälle auszulesen haben, bei denen wir mit gutem Gewissen zur Operation rathen dürfen. 